\title{
Programmed cell death 1 correlates with the occurrence and development of MG63 osteosarcoma
}

\author{
FUYOU ZHAO $^{1^{*}}$, QIONG WU $^{1^{*}}$, XIUSONG DAI $^{2^{*}}$, YUMEI LI $^{1}$, HUAIYONG GAN $^{3}$, RI WANG $^{1}$, \\ $\mathrm{JIE} \mathrm{LV}^{4}$ and YUQING $\mathrm{CHEN}^{5}$ \\ Departments of ${ }^{1}$ Medical Oncology, ${ }^{2}$ Orthopedics and ${ }^{3}$ Pathology, The First Affiliated Hospital of Bengbu Medical College; \\ ${ }^{4}$ Department of Microbiology, Bengbu Medical College; ${ }^{5}$ Department of Respiratory Diseases, \\ The Affiliated Hospital of Bengbu Medical College, Bengbu, Anhui 233000, P.R. China
}

Received February 28, 2015; Accepted June 29, 2016

DOI: $10.3892 / \mathrm{ol} .2016 .5311$

\begin{abstract}
The aim of the present study was to investigate the effect of programmed cell death 1 (PD-1) on osteosarcoma (OD) stem cells and T cells, and to determine their correlation. OS stem cells were sorted and identified from OS MG63 cells. Flow cytometry was used to detect the PD-1 expression of the OS tumor stem cell membrane surface. The expression of PD-1 mRNA was detected by reverse transcription-polymerase chain reaction (RT-PCR). MTT was used to detect the effect of PD-1 signals on T-cell proliferation. The results indicated that the cancer cells (cultured in DMEM medium containing $10 \%$ fetal bovine serum) exhibited clear proliferation within 1 week of cell culture, which showed their strong proliferation and aggressive ability. The formation of tumor cell spheres was dependent on the support of serum nutrition. The proliferation of MG63 cells in the serum culture medium was significantly higher than the number of OS cell spheres in serum-free suspension culture $(\mathrm{P}<0.05)$. Pluripotent stem cells in cancer cell spheres exhibited significantly higher cluster of differentiation 133 expression compared with the MG63 cells. The PD-1 expression levels of the cancer cell spheres was significantly increased compared with the MG63 cells, which is consistent with the results of the RT-PCR. In conclusion, the MG63 cell line possesses the features of OS stem cells. The MG63 cell line can express the certain cancer-associated cell markers. The expression of PD-1 in spheres was also increased significantly compared to the MG63 cells, which can reduce the immune function of patients and may be closely associated with the occurrence and development of tumors.
\end{abstract}

Correspondence to: Professor Yuqing Chen, Department of Respiratory Diseases, The Affiliated Hospital of Bengbu Medical College, 287 Changhuai Road, Bengbu, Anhui 233000, P.R. China E-mail: chenyuqbb@yeah.net

*Contributed equally

Key words: PD-1, MG63, osteosarcoma, stem cells, correlation

\section{Introduction}

Osteosarcoma (OS) is the most common pediatric orthopedic malignant tumor. The latest research shows that loss of regulation of the immune system is likely to be one of the most important mechanisms of occurrence for this disease (1). Programmed cell death 1 (PD-1), also known as PDCD1 or CD279, is a member of the CD28 family, and is expressed on the surface of activated T cells, B cells, dendritic cells and macrophages (2,3). Studies have shown that the resistance of PD-1 antibodies play an important role in cancer treatment and immune stimulation, and that the inhibitory effect of PD-1 on immune cells occurs mainly by inhibiting the inflammatory reaction process of the $\mathrm{T}$ cells, infection and immunity (1-3). When the PD-1 receptors of the T cell surface combine with PD-1 ligand 1 (PD-L1) and PD-L2 on its target protein chain, the intracellular phosphorylation of PD1 triggers the accumulation of phosphokinases. For example, tyrosine-protein phosphatase non-receptor type 11 inhibits T-cell receptor signaling pathways, blocking the PD-1 channel, which can strengthen the antitumor immune responses, reduce the number of inhibitory $\mathrm{T}$ cells, and enhance the activity of T-cell active factor and the organization of the anti-tumor microenvironment (4-6). By inhibiting the PD-1 channel, the activity of natural killer cells is also strengthened, prompting the generation of PD-1 B cell antibodies. PD-1 and its synergistic inhibition factors, including cytotoxic T-cell antigen 4, T-cell immune globulin, mucins area 3 , are the most common checkpoint molecules. These molecules play the role of the 'toll station', estimating extracellular signal information, and deciding whether the cell cycle or other activities in the cell can proceed. Hence, there is a close association with tumor progression $(7,8)$. At the same time, a number of studies have confirmed that cells exist in bone sarcoma tissues that are similar to stem cells and have potential for pluripotent differentiation, which is associated with the invasion and progress of the tumor $(7,8)$. The present study aimed to investigate the association between PD-1 and OS stem cells, in order to provide guidance to the use of targeted therapy in OS.

\section{Materials and methods}

Sorting and identification of OS series cancer stem cells. MG63 cells were purchased from the American Type Culture 
Collection (Manassas, VA, USA), and cultured in Dulbecco's modified Eagle's medium (DMEM)/F12 medium containing $10 \%$ fetal bovine serum at room temperature and $5 \% \mathrm{CO}_{2}$ for 10 generations. To form OS spheres ( $>50$ cells), the MG63 cells were cultured at room temperature in DMEM/F12 serum free medium with epidermal growth factor (EGF; $10 \mathrm{ng} / \mathrm{ml}$ ), basic fibroblast growth factor (bFGF; $10 \mathrm{ng} / \mathrm{ml}$ ) and $\mathrm{N} 2$ additives $(20 \% \mathrm{~N} 2)$ in a low adhesion cell culture plate suspension for 7-12 days. The OS cells were removed, and added to the serum-free suspension medium, and then it was observed whether cells were able to reform spheres. The OS cell sphere vaccinate was added into a culture environment (room temperature) containing serum OS and growth was observed. Bone sarcoma MG63 cells and OS cells were collected subsequent to being cultured in a serum-free suspension for 7-10 days. MG63 cells $\left(1 \times 10^{5}\right)$ and stem cells in OS cell spheres were analyzed and compared for the expression of associated surface markers, such as CD133 (examined using rabbit anti-human CD133 polyclonal antibody; dilution, 1:3,000; catalog no., ab19898; Abcam, Cambridge, MA, USA), as determined by flow cytometry (FCM). Bone sarcoma MG63 cells and OS cells were collected subsequent to being cultured in a serum-free suspension for 7-10 days, and then the growth situation of the MG63 cells and OS cell spheres was detected following incubation with EGF $(10 \mathrm{ng} / \mathrm{ml})$, bFGF (10 $\mathrm{ng} / \mathrm{ml})$ and $\mathrm{N} 2$ additives (20\% N2) in DMEM/F12 serum-free medium from days 1-7, and the growth curve was depicted.

FCM detects the expression of PD-1 on the membrane surface of OS tumor stem cells. The OS cancer stem cells were stained with phycoerythrin (PE)-labeled PD- 1 at $4^{\circ} \mathrm{C}$ for $20 \mathrm{~min}$ in the dark. PE-labeled mouse anti-human immunoglobulin $\mathrm{G}$ was used as an isotype control, detecting the expression of PD-1 protein in the cell membrane. The cells were flushed using $0.1 \%$ calf serum phosphate-buffered saline solution at $48^{\circ} \mathrm{C}$, incubated and then added to the 10 -ml antibodies. FACS Vantage (BD Biosciences, Franklin Lakes, NJ, USA) and Epics XL (BD Biosciences) instruments were used for data analysis. PE-conjugated mouse anti-human CD133 (clone AC133/1; dilution, 1:3,000; catalog no., 130-098-826; Miltenyi Biotec, Bergisch Gladbach, Germany), mouse anti-human CD34 (clone AC136; dilution, 1:3,000; catalog no., 130-098-140; Miltenyi Biotec), mouse anti-human CD117 (clone A3C6E2; dilution, 1:3,000; catalog no., 130-098-212; Miltenyi Biotec), mouse anti-human CD24 (clone 32D12; dilution, 1:3,000; catalog no., 130-098-861; Miltenyi Biotec), mouse anti-human CD90 (clone DG3; dilution, 1:3,000; catalog no., 130-097-932; Miltenyi Biotec), mouse anti-human CD29 (clone TS2/16; dilution, 1:3,000; catalog no., 130-101-275; Miltenyi Biotec), monoclonal mouse anti-human CD44 (dilution, 1:3,000; catalog no., ab46793; Abcam), monoclonal mouse anti-human CD105 (dilution, 1:3,000; ab53321; Abcam) and monoclonal mouse anti-human CD271 (dilution, 1:3,000; catalog no., ab157333; Abcam) antibodies were used. Isolated cells were stained on ice for $30 \mathrm{~min}$.

Reverse transcription-polymerase chain reaction (RT-PCR). The OS cell spheres were collected using TRIzol to extract total mRNA, according to the manufacturer's instructions in the Takara retrovirus kit (Takara Bio Inc., Otsu, Japan). mRNA $(2 \mu \mathrm{g})$ was reverse-transcribed into cDNA by using $1 \mu \mathrm{l}$ reverse transcription reaction product with $20 \mu \mathrm{l}(10 \mathrm{pmol})$ primers using a PCR machine (5331 MasterCycler; Eppendorf, Hamburg, Germany). All reverse transcription reaction reagents were part of the RevertAid First Strand cDNA Synthesis kit (Thermo Fisher Scientific, Inc., Waltham, MA, USA). Reagents for PCR, including DNA polymerase, dNTPs, primers, buffer and DNase were part of the QuantiTect SYBR Green PCR kit (Qiagen GmbH, Hilden, Germany). The amplification protocols were as follows: $94^{\circ} \mathrm{C}$ for $30 \mathrm{sec}$, followed by annealing at $60^{\circ} \mathrm{C}$ for $30 \mathrm{sec}$ and extension at $72^{\circ} \mathrm{C}$ for $60 \mathrm{sec}$ for a total of 30 cycles. $\beta$-actin was used as a loading control. The primer sequences were as follows: Oct3/4 forward, 5'-TGGAGAAGG AGAAGCTGGAGCAAAA-3' and reverse, 5'-GGCAGAGGT CGTTTGGCTGAATAGACC-3'; nanog forward, 5'-TCCTCC TCTTCCTCTATACTAAC-3' and reverse, 5'-CCCACAATC ACAGGCATAG-3'); nestin forward, 5'-GAGGACCAGAGT ATTGTGAGAC-3' and reverse, 5'-CACAGTGGTGCTTGA GTTTC-3'; MDR1 forward, 5'-TTCAAACTTGTCACAATG CAGACAGCAGGA-3' and reverse, 5'-GGTTGCAGGCCT CCATTTATAATGGCACAA-3'; PD-1 forward, 5'-CCCAAG GCGCAGATCAA-3' and reverse, 5'-GCACTTCTGCCCTTC TCTCTGT-3'; CD133 forward, 5'-GCACTCTATACCAAA GCGTCAA-3' and reverse, 5'-CTCCCATACTTCTTAGTT TCCTCA-3'; and $\beta$-actin forward, 5'-GCGGGAAATCGT GCGTGACATT-3' and reverse, 5'-GGCAGATGGTCGTTT GGCTGAATA-3'. The PCR reaction was repeated at least 6 times.

Following electrophoresis on $1.4 \%$ agarose gels (with ethidium bromide), the gel images of each PCR product were digitally captured with a charge-coupled device camera and analyzed with the ImageJ version 1.45 (imagej.nih.gov/ij/).

Statistical analysis. SPSS 16.0 software (SPSS Inc., Chicago, IL, USA) was used for data analysis. Student's t-test was used for comparative analysis between two groups. Data is presented as the mean \pm standard deviation. $\mathrm{P}<0.05$ was used to indicate a statistically significant difference.

\section{Results}

Proliferation of OS cells. The majority of cells were fusiform or polygon in shape, with no contact inhibition. As shown in Fig. 1A and B, obvious cancer cell proliferation was detected within 1 week, indicating their strong proliferation and aggressive ability. The MG-63 cells were either trained in medium containing $10 \%$ fetal bovine serum or in serum-free medium for 10 days to form tumor cell spheres (Fig. 1C and D). The former number of cancer cells and the volume of tumor formation were significantly increased. If we remove the spheres of the OS cells and add them to the serum-free suspension culture medium, there was no obvious formation of tumor cell spheres 12 days later. With the OS cell spheres vaccinated to a serum-containing culture environment, marked proliferation of cancer cells and formation of tumor cell spheres was evident, and at the same time around 12 days, the cancel cells were spreading and invading their surroundings (Fig. 1E and F). Counting MG63 cells and OS cell spheres cultured for 1-7 days within serum-free suspension showed similar results to those observed 

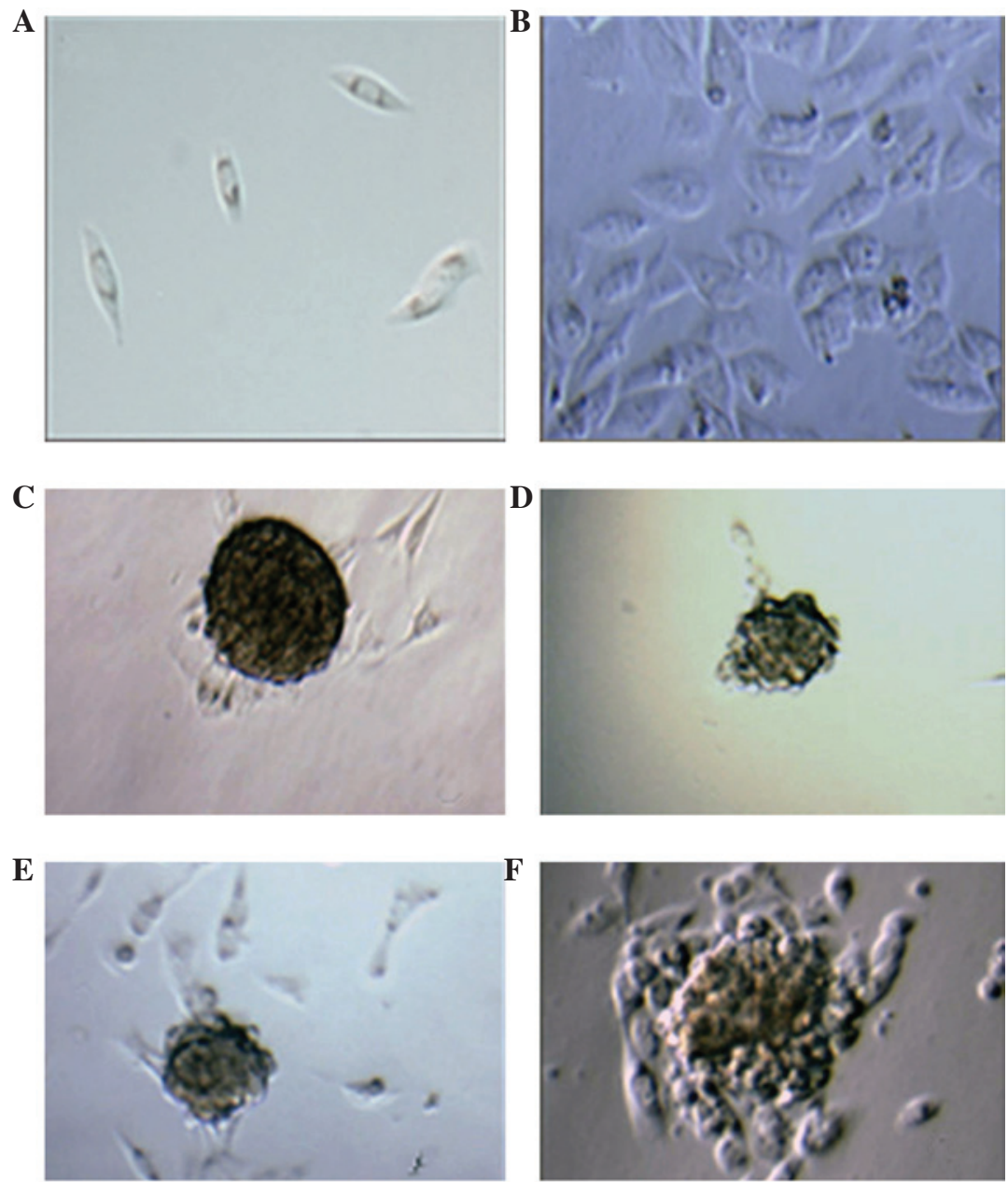

Figure 1. Proliferation and morphology of osteosarcoma cells. (A and B) Microscopy images of cancer cell growth in serum medium at (A) 1 and (B) 7 days (x40 magnification). (C) Cancer cell growth in 10\% fetal bovine serum at 10 days showing cell sphere formation. (D) Cancer cell sphere formation after 10 days without serum. (E) Cancer cell proliferation when osteosarcoma cell spheres are vaccinated into a serum cultured environment and become tumor cells spheres at 7 days. (F) Cancer cells could be observed visibly spreading and invading their surroundings at $~ 12$ days. Magnification, x200.

by microscopy, with the proliferation number of MG63 cells in the serum culture medium being significantly higher than the cell spheres $(\mathrm{P}=0.031,0.021,0.019,0.014,0.015,0.034$ and 0.045 for days 1 to 7, respectively, for the MG63 cells compared with the osteosarcoma cell spheres; Fig. 2).

Detection of stem cell surface markers by FCM. Surface marker expression of ectomesenchymal stem cells (CD105, CD90, CD44, CD29), hematopoietic stem cells (CD34, CD133) and epithelial stem cells (CD24) was determined by flow cytometry. The study failed to detect CD34, CD31 and CD105 expression in the MG63 cells and the cancer spheres. The expression of CD90 and CD44 was significantly increased in the two cell types. CD29 expression in the cancer cell spheres was higher than that in the MG63 cells, while CD24 expression was highest in the MG63 cells. The expression level of the stem cell marker CD133 in the spheres was significantly higher than that in the MG63 cells, which is consistent with the results of the RT-PCR. Meanwhile, the expression of PD-1 in the cancer cell spheres was increased compared to the MG63 cells (Fig. 3).

Detection of mRNA expression in cancer cells by RT-PCR. mRNA analysis showed that the specific pluripotent stem cell

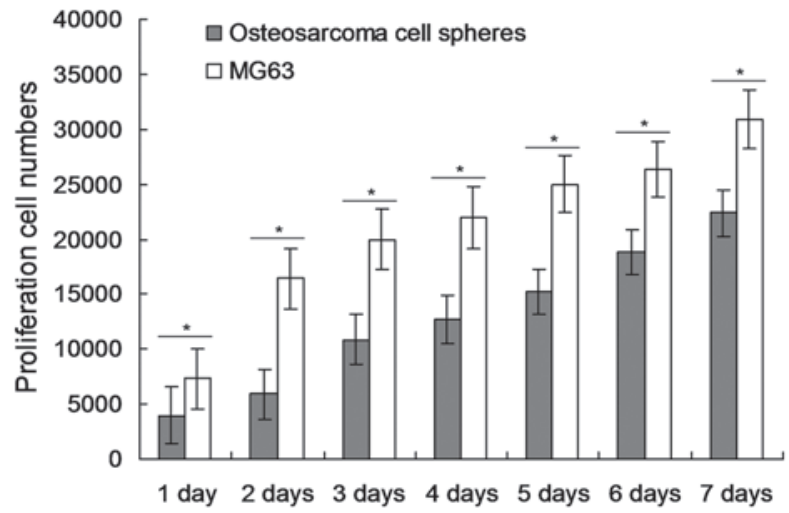

Figure 2. Proliferation of MG63 cells and osteosarcoma cell spheres cultured within serum-free suspension for $1-7$ days. ${ }^{*} \mathrm{P}<0.05$.

marker CD133 and the embryonic stem cell sphere markers octamer-binding transcription factor (OCT) $3 / 4$, nestin and nanog were markedly enhanced in the cancer cell spheres compared with the MG63 cells. The expression of PD-1 mRNA in the cancer cell spheres was also increased compared to the MG63 cells (Fig. 4). 


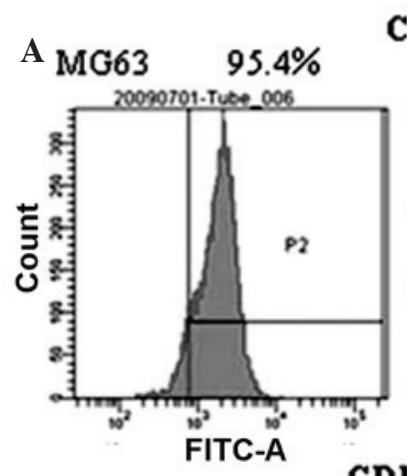

CD44

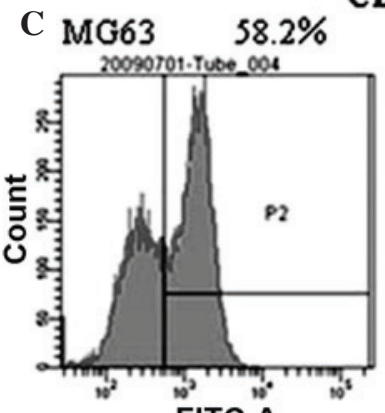

FITC-A

E MG63 $1.42 \%$

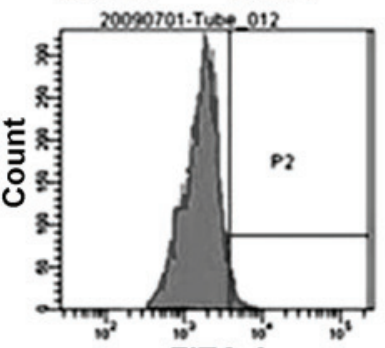

FITC-A

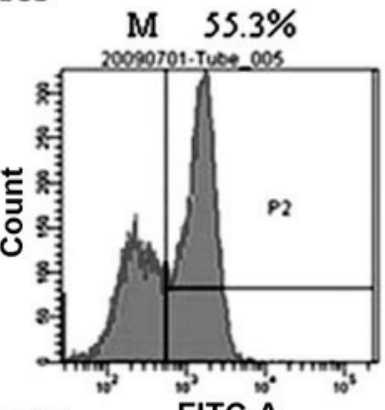

FITC-A

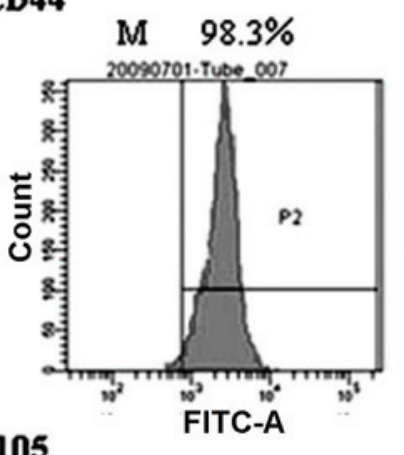

B MG63 $97.2 \%$

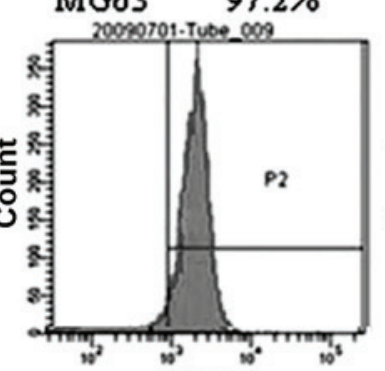

FITC-A
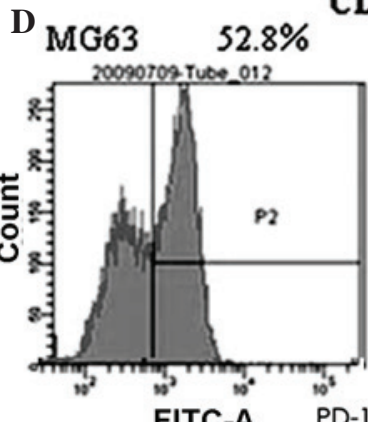

FITC-A PD-

F $\quad$ MG63 $94.6 \%$

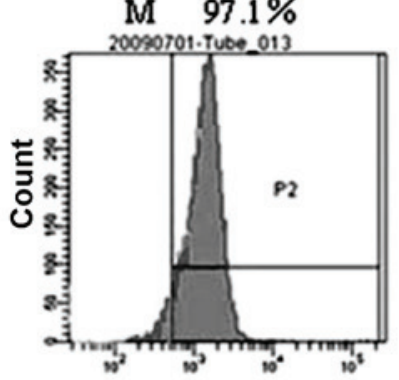

FITC-A

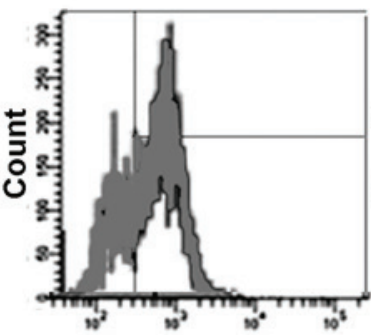

FITC-A
CD90

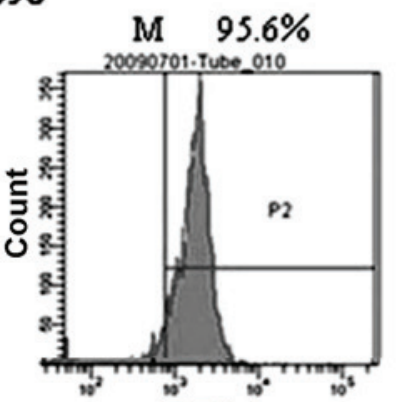

FITC-A

CD24

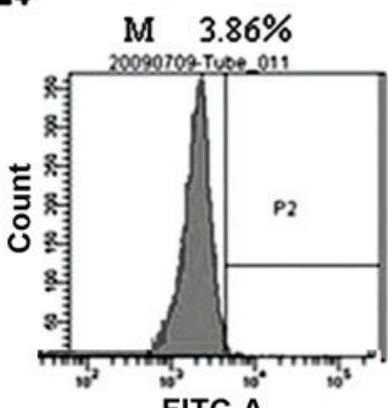

FITC-A

M $\quad 93.3 \%$

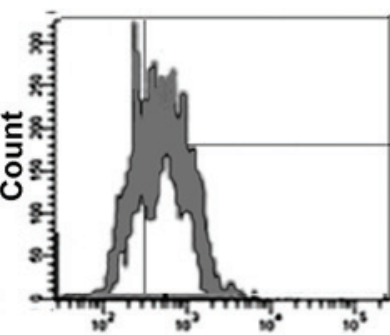

FITC-A

Figure 3. Detection of stem cell surface markers by flow cytometry, and the expression of PD-1. CD, cluster of differentiation; PD-1, programmed cell death 1; FITC, fluorescein isothiocyanate. M, osteosarcoma cell spheres.

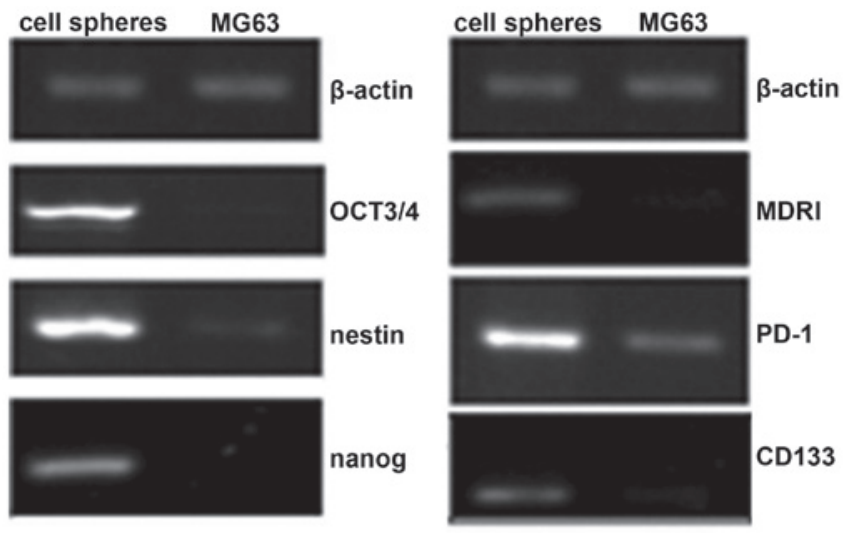

Figure 4. Detection of mRNA expression in cancer cells by reverse transcription-polymerase chain reaction. OCT, octamer-binding transcription factor; CD, cluster of differentiation; MDR1, multidrug resistance 1; PD-1, programmed cell death 1 .

\section{Discussion}

Although they exhibit allobiosis, MG63 cells are not monoclonal. This is believed to be important to cytological study from the point of the purification of monoclonal cells of cell lines $(9,10)$. In order to obtain monoclonal stem cell samples from MG63 cells, the cloned cells densely distributed in a round or oval shape were selected, which have the ability to form tumors. However, the cultured monoclonal cells are not able to maintain specific characteristics. Therefore, the cells used for each experiment are derived from the same line in the present study. Contrasting with the osteosarcoma cells spheres, the expression level of cancer stem cell-associated genes in the MG63 cells was relatively low. In total, $>90 \%$ of the cells in the cancer cell spheres expressed the pluripotent stem cell marker CD133, while the expression level in the MG63 cells was low.

The migration, invasion, conglutination and growth analyses of cancer cells are the most commonly used methods to judge cytoactivity. These methods can reflect the fact that the tumor leaves the primary tumor extracellular matrix (11-15). In the present study, it was found that the reproduction rate of the tumor cells was relatively low in the early passage. With the increase of passages, the reproduction rate also increased. However, there was no clear difference in the invasion and transfer ability of the cells. The analysis of the cell cycle showed that the percentage of cells in the G0/G1 phase was relatively high. This means that the cloning ability was strong. 
Cancer stem cells mainly express markers of mesenchymal stem cells (CD29, CD44, CD105 and CD45), and express a number of genes derived from the mesoderm, ectoderm and endoderm $(16,17)$. Tumor spheres express markers of embryonic stem cells (OCT3/4, nestin and nanog) (16). Further studies have shown that various stem cell markers are often observed in the early formation stage of tumor spheres; with an increase in the volume of the sphere and an increase in the number of cells, the expression of these various markers decreases, and so the balls of cells become more specialized (and diversified), and each tumor cell becomes more homogenous $(16,17)$. Therefore, as they are growing, the original tumor cells are making the transition towards tumor heterogeneity, i.e. clumps of different cancerous cells are growing that originated from the tumor sphere during its early formation. Heterogeneity that is observed between tumor cells is the result of differences in the stem cells from which they originated $(16,17)$. Oct- $3 / 4$ is transcription factor of the POU family. At the early stage, the Oct-3/4 is mainly expressed in the endoderm, and associated with the pluripotent maintenance of embryonic stem cells, with the growth of the embryo, its expression level gradually decreases (18-20). The gene expression of nanog is not commonly found in somatic cells, and the activation of the gene is vital for the control of self-renewal and the pluripotency of embryonic stem cells (21). In the present study, the expression level of the nanog genes in the cancer cells was markedly increased, indicating that cancer cell spheres, as in embryonic stem cells, exhibit equal multidirectional differentiation potential. ОСТ $3 / 4$, nanog and nestin are associated with cell differentiation and self-renewal (21). If such a protein in tumor stem cell samples has the same effect, it will become the ideal target therapy for OS (21).

At present, a number of studies have confirmed that PD-1 is a synergistic inhibitory receptor, which can regulate $\mathrm{T}$ cell proliferation $(12,22)$. The antigen-stimulated high expression of PD-1 on the $\mathrm{CD} 8^{+} \mathrm{T}$-cell surface is associated with $\mathrm{T}$-cell depletion. The cytokine secretion of $\mathrm{CD} 8^{+} \mathrm{T}$ cells, plus interleukin 2, tumor necrosis factor- $\alpha$, interferon- $\gamma$ and other cell factors are gradually lost. PD $-1^{+} \mathrm{CD} 8^{+} \mathrm{T}$ cells are found in tumor-infiltrating or chronic virus-infected lymphocytes (22). The function of PD- 1 in CD4 ${ }^{+} \mathrm{T}$ cells remains unclear at present. Certain studies have shown that PD-1 can limit the degree of $\mathrm{CD}^{+} \mathrm{T}$-cell aggregation following immunostimulation. The $\mathrm{CD} 4^{+} \mathrm{T}$-cell regulating action of $\mathrm{PD}-1$ varies in different illnesses. The expression of PD- 1 in $\mathrm{CD}^{+}{ }^{+} \mathrm{T}$ cells may be closely associated with the progression of numerous diseases $(23,24)$. The PD-1 expression of $\mathrm{CD}^{+}$and $\mathrm{CD} 8^{+}$ $\mathrm{T}$ cells in OS was increased significantly compared with MG63 cells. The PD-1 level is higher with tumor metastasis, in advanced tumors or with merged pathological fracture $(23,24)$. RT-PCR and flow cytometry have confirmed that the expression of PD-1 is markedly increased with the proliferation of tumor cells and enhanced invasiveness. The expression of PD-1 is closely associated with the occurrence and progression of tumors $(23,24)$.

The MG63 cell line possesses the feature of OS stem cells. The MG63 cell line can express the corresponding cell markers. The expression of PD-1 is also increased significantly, which can reduce the immune functions of affected patients and is closely associated with the occurrence and development of tumors.

\section{Acknowledgements}

This study was supported by the Project of Anhui Province for Excellent Young Talents in Universities (grant no. 2013SQRL049ZD) and the Project of Anhui Provincial Department of Health (grant no. KJ2012Z251).

\section{References}

1. Bai X, Meng H, Ma L and Guo A: Inhibitory effects of evodiamine human osteosarcoma cell proliferation and apoptosis. Oncol Lett 9: 801-805, 2015.

2. Fleuren ED, Versleijen-Jonkers YM, Boerman OC and van der Graaf WT: Targeting receptor tyrosine kinases in osteosarcoma and Ewing sarcoma: Current hurdles and future perspectives. Biochim Biophys Acta 1845: 266-276, 2014.

3. Botter SM, Neri D and Fuchs B: Recent advances in osteosarcoma. Curr Opin Pharmacol 16: 15-23, 2014.

4. Siewe B, Wallace J, Rygielski S, Stapleton JT, Martin J, Deeks SG and Landay A: Regulatory B cells inhibit cytotoxic T lymphocyte (CTL) activity and elimination of infected CD4 T cells after in vitro reactivation of HIV latent reservoirs. PLoS One 9: e92934, 2014.

5. Xu P, Chen YJ, Chen H, Zhu XY, Song HF, Cao LJ and Wang XF: The expression of programmed death-1 in circulating $\mathrm{CD}^{+}$and $\mathrm{CD} 8^{+} \mathrm{T}$ cells during hepatitis $\mathrm{B}$ virus infection progression and its correlation with clinical baseline characteristics. Gut Liver 8: 186-195, 2014.

6. Philip NH, Dillon CP, Snyder AG, Fitzgerald P, Wynosky-Dolfi MA, Zwack EE, Hu B, Fitzgerald L, Mauldin EA, Copenhaver AM, et al: Caspase- 8 mediates caspase- 1 processing and innate immune defense in response to bacterial blockade of NF-kB and MAPK signaling. Proc Natl Acad Sci USA 11: 7385-7590, 2014.

7. Pedoeem A, Azoulay-Alfaguter I, Strazza M, Silverman GJ and Mor A: Programmed death-1 pathway in cancer and autoimmunity. Clin Immunol 153: 145-152, 2014.

8. Notaro A, Sabella S, Pellerito O, Di Fiore R, De Blasio A, Calvaruso $G$ and Giuliano M: Involvement of PAR-4 in cannabinoid-dependent sensitization of osteosarcoma cells to TRAIL-induced apoptosis. Int J Biol Sci 10: 466-478, 2014.

9. Oba J, Nakahara T, Abe T, Hagihara A, Moroi Y and Furue M: Expression of programmed death receptor ligand 1 in melanoma may indicate tumor progression and poor patient survival. J Am Acad Dermatol 70: 954-956, 2014.

10. Mittendorf EA, Philips AV, Meric-Bernstam F, Qiao N, Wu Y, Harrington S, Su X, Wang Y, Gonzalez-Angulo AM, Akcakanat A, et al: PD-L1 expression in triple-negative breast cancer. Cancer Immunol Res 2: 361-370, 2014.

11. Joseph RW, Parasramka M, Eckel-Passow JE, Seri D, Wu K, Jiang L, Kalari K, Thompson RJ, Huu Ho T, Castle EP, et al: Inverse association between programmed death ligand 1 and genes in the VEGF pathway in primary clear cell renal cell carcinoma. Cancer Immunol Res 1: 378-385, 2013.

12. Macfarlane AW IV, Jillab M, Plimack ER, Hudes GR, Uzzo RG, Litwin S, Dulaimi E, Al-Saleem T and Campbell KS: PD-1 expression on peripheral blood cells increases with stage in renal cell carcinoma patients and is rapidly reduced after surgical tumor resection. Cancer Immunol Res 2: 320-331, 2014.

13. Flies DB, Han X, Higuchi T, Zheng L, Sun J, Ye JJ and Chen L: Coinhibitory receptor PD-1H preferentially suppresses $\mathrm{CD}^{+}$ T cell-mediated immunity. J Clin Invest 124: 1966-1975, 2014.

14. Barker CA and Postow MA: Combinations of radiation therapy and immunotherapy for melanoma: A review of clinical outcomes. Int J Radiat Oncol Biol Phys 88: 986-997, 2014.

15. Tosti G, Cocorocchio E and Pennacchioli E: Anti-cytotoxic T lymphocyte antigen-4 antibodies in melanoma. Clin Cosmet Investig Dermatol 6: 245-256, 2013.

16. Feng D, Yang X, Li S, Liu T, Wu Z, Song Y, Wang J, Gao W, Huang Q, Huang W, et al: Cytotoxic T-lymphocyte antigen-4 genetic variants and risk of Ewing's sarcoma. Genet Test Mol Biomarkers 17: 458-463, 2013.

17. Liu Y, He Z, Feng D, Shi G, Gao R, Wu X, Song W and Yuan W: Cytotoxic T-lymphocyte antigen-4 polymorphisms and susceptibility to osteosarcoma. DNA Cell Biol 30: 1051-1055, 2011.

18. Wang W, Wang J, Song H, Liu J, Song B and Cao X: Cytotoxic T-lymphocyte antigen- $4^{+} 49 \mathrm{G} / \mathrm{A}$ polymorphism is associated with increased risk of osteosarcoma. Genet Test Mol Biomarkers 15: 503-506, 2011. 
19. Yang S, Wang C, Zhou Y, Sun G, Zhu D and Gao S: Cytotoxic T-lymphocyte antigen-4 polymorphisms and susceptibility to Ewing's sarcoma. Genet Test Mol Biomarkers 16: 1236-1240, 2012.

20. Shang Y, Li Z, Li H, Xia H and Lin Z: TIM-3 expression in human osteosarcoma: Correlation with the expression of epithelial-mesenchymal transition-specific biomarkers. Oncol Lett 6: 490-494, 2013.

21. Ramsay AG: Immune checkpoint blockade immunotherapy to activate anti-tumour T-cell immunity. Br J Haematol 162: 313-325, 2013.
22. Ott PA, Hodi FS and Robert C: CTLA-4 and PD-1/PDL1 blockade: New immunotherapeutic modalities with durable clinical benefit in melanoma patients. Clin Cancer Res 19: 5300-5309, 2013

23. Okazaki T, Chikuma S, Iwai Y, Fagarasan S and Honjo T: A rheostat for immune responses: The unique properties of PD-1 and their advantages for clinical application. Nat Immunol 14: 1212-1218, 2013

24. Pan XC, Li L, Mao JJ, Yao W, Zheng JN, Liu M and Fu JJ: Synergistic effects of soluble PD-1 and IL-21 on antitumor immunity against $\mathrm{H} 22$ murine hepatocellular carcinoma. Oncol Lett 5: 90-96, 2013. 